\title{
REGULARIZABILITY OF ILL-POSED PROBLEMS AND THE MODULUS OF CONTINUITY
}

\author{
RADU IOAN BOT,, BERND HOFMANN, AND PETER MATHÉ \\ Dedicated to Ulrich Tautenhahn on the occasion of his 60th birthday
}

\begin{abstract}
The regularization of linear ill-posed problems is based on their conditional well-posedness when restricting the problem to certain classes of solutions. Given such class one may consider several related real-valued functions, which measure the wellposedness of the problem on such class. Among those functions the modulus of continuity is best studied. For solution classes which enjoy the additional feature of being star-shaped at zero, the authors develop a series of results with focus on continuity properties of the modulus of continuity. In particular it is highlighted that the problem is conditionally well-posed if and only if the modulus of continuity is right-continuous at zero. Those results are then applied to smoothness classes in Hilbert space. This study concludes with a new perspective on a concavity problem for the modulus of continuity, recently addressed by two of the authors in Some note on the modulus of continuity for ill-posed problems in Hilbert space, 2011.
\end{abstract}

\section{INTRODUCTION}

We shall consider linear ill-posed problems, given in the form

$$
y^{\delta}=A x+\delta \xi
$$

where $A: X \rightarrow Y$ denotes an injective and bounded linear operator acting between Banach spaces $X$ and $Y$. If the range $\mathcal{R}(A) \subset Y$ is non-closed, then the problems (1) are ill-posed, meaning that the inverse mapping $A^{-1}: \mathcal{R}(A) \subset Y \rightarrow X$ is not bounded and hence not continuous. However, one may restrict a problem as posed in (1) by introducing the $a$ priori information that $x \in M \subseteq X$, for a non-empty subset $M$. By doing so we may study the following questions.

Question 1. Does the mapping $A: M \rightarrow Y$ have a bounded inverse?

If this is the case, then the mapping $\Omega_{M}:[0,+\infty) \rightarrow[0,+\infty]$, given as

$$
\Omega_{M}(\delta):=\sup \left\{\left\|x-x^{\prime}\right\|: x, x^{\prime} \in M,\left\|A x-A x^{\prime}\right\| \leq \delta\right\},
$$

is of interest. The above function $\Omega_{M}$ is analyzed in various studies, and we mention [7, § 2.3] for a monograph.

Question 2. Is the problem (1) regularizable on $M$ in the sense of Tikhonov?

2010 Mathematics Subject Classification. 47A52, secondary: 65J20. 
This question is related to the reconstruction of $x$ from noisy data, and we introduce the error criterion. If $S$ is any mapping of the form

$$
y^{\delta} \in Y \rightarrow S\left(y^{\delta}\right) \in X
$$

then we let

$$
e(S, x, \delta):=\sup _{y^{\delta}:\left\|A x-y^{\delta}\right\| \leq \delta}\left\|S\left(y^{\delta}\right)-x\right\|, \quad \delta>0
$$

and the corresponding uniform error of any reconstruction $S$ on the set $M$ will be given by

$$
e(S, M, \delta):=\sup _{x \in M} e(S, x, \delta), \quad \delta>0
$$

By regularizability on $M$ in the sense of Tikhonov we mean that there is a family $R_{\delta}$ of reconstructions such that for each $x \in M$ we have that

$$
e\left(R_{\delta}, x, \delta\right) \longrightarrow 0 \quad \text { as } \delta \rightarrow 0
$$

The question whether a problem is regularizable received attention very early in the analysis of ill-posed problems (for an early study we refer to [11], and we also mention the monograph [1, Chapt. 1]). If the above is the case then we call the restriction of $A^{-1}$ on

$$
A(M):=\{z \in Y: z=A x, x \in M\}
$$

regularizable on $A(M)$. In addition we may ask for uniform regularizability of the problem (1) on the set $M$, thus asking whether a family $R_{\delta}$ of reconstructions exists for which $e\left(R_{\delta}, M, \delta\right) \rightarrow 0 \quad$ as $\delta \rightarrow 0$.

While the first question studies intrinsic continuity features of the problem, the latter question asks for the ability to find reconstruction methods, which are capable to recover $x$ based on noisy data $y^{\delta}$ as $\delta$ is getting smaller. In this study we will not address the regularizability problem in the sense of Tikhonov. Instead we analyze uniform regularizability for classes $M$.

\section{Modulus of COntinuity}

For a variety of classes of subsets $M$ of $X$ that contain the zero element, instead of the function $\Omega_{M}$ from (2) one can consider the function $\omega_{M}:[0,+\infty) \rightarrow[0,+\infty]$, defined as

$$
\omega_{M}(\delta):=\sup \{\|x\|: x \in M,\|A x\| \leq \delta\},
$$

usually called modulus of continuity. Clearly, for $0 \in M$ we have that $\omega_{M}(\delta) \leq \Omega_{M}(\delta)$ for all $\delta \geq 0$. The modulus of continuity is obviously a non-decreasing function of $\delta$. 
2.1. Elementary properties. The following elementary properties are easily verified.

Lemma 1. Let $M, N \subseteq X$ be two subsets. Then for all $\delta \geq 0$ it holds

(i) $\omega_{M \cup N}(\delta)=\max \left\{\omega_{M}(\delta), \omega_{N}(\delta)\right\}$.

(ii) $\omega_{M}(\delta)=\omega_{-M}(\delta)$.

In particular, we have that

$$
\omega_{M \cup-M}(\delta)=\omega_{M}(\delta) .
$$

Below we shall confine ourselves to sets $M$ from the following class of sets.

Definition 1. A subset $M \subseteq X$ is said to be star-shaped at zero if $0 \in M$ and if $x \in M$ implies $\alpha x \in M$ for all $0 \leq \alpha \leq 1$.

Lemma 2. Suppose that $M$ is star-shaped at zero. Then for all $\delta \geq 0$ and all $C \geq 1$ it holds

$$
\omega_{M}(C \delta) \leq C \omega_{M}(\delta) \text { and } \Omega_{M}(C \delta) \leq C \Omega_{M}(\delta) .
$$

In particular, we have that $\omega_{M}(t) / t \leq \omega_{M}(s) / s$ as well as $\Omega_{M}(t) / t \leq \Omega_{M}(s) / s$ whenever $0<s \leq t$.

Proof. For $C \geq 1, \delta \geq 0$, taking into account Definition 1 with $\alpha:=1 / C$, it holds

$$
\begin{aligned}
\omega_{M}(C \delta) & =\sup \{\|x\|: x \in M,\|A x\| \leq C \delta\} \\
& =C \sup \left\{\left\|\frac{1}{C} x\right\|: \frac{1}{C} x \in \frac{1}{C} M,\left\|A \frac{1}{C} x\right\| \leq \delta\right\} \\
& =C \sup \left\{\|z\|: z \in \frac{1}{C} M,\|A z\| \leq \delta\right\} \\
& =C \omega_{\frac{1}{C} M}(\delta) \leq C \omega_{M}(\delta) .
\end{aligned}
$$

The assertion for $\Omega_{M}$ follows, by taking into consideration that $\Omega_{M}=\omega_{M-M}$.

We summarize the above elementary findings.

Proposition 1. Suppose that the set $M \subseteq X$ is star-shaped at zero. Then

(i) the mapping $\omega_{M}$ is non-decreasing, $\omega_{M}(0)=0$, and the mapping $\delta \rightarrow \omega_{M}(\delta) / \delta$ is non-increasing on $(0,+\infty)$.

(ii) Likewise the mapping $\Omega_{M}$ is non-decreasing, $\Omega_{M}(0)=0$, and the mapping $\delta \rightarrow \Omega_{M}(\delta) / \delta$ is non-increasing on $(0,+\infty)$.

These elementary properties have immediate consequences for the continuity properties of both $\omega_{M}$ and $\Omega_{M}$.

\subsection{Modulus functions.}

Definition 2. We agree to call a mapping $f:[0,+\infty) \rightarrow[0,+\infty]$ a modulus function if it is non-decreasing, $f(0)=0$, and $t \rightarrow f(t) / t$ is non-increasing on $(0,+\infty)$. It is called proper if for some $t>0$ it has a finite value $f(t)<+\infty$. 
With this notion both the functions $\omega_{M}$ and $\Omega_{M}$ are modulus functions provided that the set $M$ is star-shaped at zero.

Proposition 2. Let $f$ be any proper modulus function. Then

(i) the values $f(t)$ are finite for every $t>0$.

(ii) If $f\left(t_{0}\right)=0$ for some $t_{0}>0$ then $f(t)=0$ for all $t \geq 0$.

(iii) The mapping $f$ is continuous on $(0,+\infty)$, and

(iv) for every pair $t_{1}, t_{2}>0$ we have that

$$
f\left(t_{1}+t_{2}\right) \leq f\left(t_{1}\right)+f\left(t_{2}\right) .
$$

Proof. Suppose that $f$ is finite at $t_{0}>0$. Then it is finite for every $0<t \leq t_{0}$ by monotonicity. Also, if $t>t_{0}$ then $f(t) / t \leq f\left(t_{0}\right) / t_{0}<+\infty$, which proves the assertion (ii).

For the second assertion (ii), suppose that $f\left(t_{0}\right)=0$, and $t_{0}>0$. Then, due to monotonicity we have that $f(t)=0,0 \leq t \leq t_{0}$. For any $t_{1}>t_{0}$ we see that $0 \leq f\left(t_{1}\right)=$ $f\left(\frac{t_{1}}{t_{0}} t_{0}\right) \leq \frac{t_{1}}{t_{0}} f\left(t_{0}\right)=0$.

For proving (iii) let $t>0$ be any real number. We first prove right continuity of $f$ at $t$. Let $t_{n} \searrow t$. Then $1 \leq f\left(t_{n}\right) / f(t) \leq t_{n} / t \rightarrow 1$. Similarly, if $t_{n} \nearrow t$ then $1 \leq f(t) / f\left(t_{n}\right) \leq$ $t / t_{n} \rightarrow 1$, which proves the continuity of $f$ at $t>0$.

The proof of the sub-additivity in item (iv) is well-known, and we recall this here for convenience. Plainly, $t_{1}, t_{2} \leq t_{1}+t_{2}$ such that

$$
\begin{aligned}
f\left(t_{1}+t_{2}\right) & =t_{1} \frac{f\left(t_{1}+t_{2}\right)}{t_{1}+t_{2}}+t_{2} \frac{f\left(t_{1}+t_{2}\right)}{t_{1}+t_{2}} \\
& \leq t_{1} \frac{f\left(t_{1}\right)}{t_{1}}+t_{2} \frac{f\left(t_{2}\right)}{t_{2}}=f\left(t_{1}\right)+f\left(t_{2}\right),
\end{aligned}
$$

and this completes the proof of the proposition.

Remark 1. Proposition2 (iv yields that $\left|f\left(t_{1}\right)-f\left(t_{2}\right)\right| \leq f\left(\left|t_{1}-t_{2}\right|\right), t_{1}, t_{2} \geq 0$. Therefore, proper modulus functions are sub-additive, and continuous at every $t>0$.

Within the classical context, the modulus of continuity, say $\omega$, of a real function on a bounded interval is non-decreasing, $\omega(0)=0$, and sub-additive, see e.g. [8, $\S$ 6.1]. Therefore any such function is called modulus of continuity if, in addition, it is continuous at zero.

Corollary 1. A proper modulus function is continuous on $[0,+\infty)$ if and only if it is right-continuous at zero.

We conclude this subsection with gathering more, and important, properties of modulus functions, we refer to [8, Lemma 6.1.4].

Proposition 3. For every proper modulus function $f$ which is right-continuous at zero there is a concave right-continuous proper modulus function $f_{*}$ with

$$
f(t) \leq f_{*}(t) \leq 2 f(t), \quad t>0 .
$$

The constant 2 cannot be improved, in general.

We finally mention the following result. 
Corollary 2. If a proper modulus function $f$ does not vanish identically then it tends to zero at most linearly, i.e., $t=\mathcal{O}(f(t))$ as $t \searrow 0$.

Proof. Suppose that $f$ does not vanish identically and that it is finite for $t_{0}>0$. According to Proposition 2 2i], $f$ is finite on $[0,+\infty)$. Then the assertion is immediate from the fact that $f(t) / t$ is non-increasing, which implies that for all $0<t \leq t_{0}$ we have that $f(t) \geq \frac{f\left(t_{0}\right)}{t_{0}} t$.

2.3. Conditional well-posedness. The right-continuity at zero of the functions $\omega_{M}$ and $\Omega_{M}$ is intimately related to the continuity of the inverse $A^{-1}$, when considered as acting from $A(M) \subset Y$ to $X$. Indeed, we have the following proposition.

Proposition 4. Let $M \subseteq X$ be star-shaped at zero. Then the modulus $\omega_{M}$ is rightcontinuous at zero if and only if the mapping $A^{-1}$ restricted to $A(M)$ is continuous at zero.

Proof. One has that $\omega_{M}$ is right-continuous at zero if and only if

$$
\begin{gathered}
\forall \varepsilon>0 \exists \bar{\delta}>0 \text { such that } \forall \delta<\bar{\delta} \text { it holds } \omega_{M}(\delta)<\varepsilon \Leftrightarrow \\
\forall \varepsilon>0 \exists \bar{\delta}>0 \text { such that } \forall \delta<\bar{\delta} \forall y \in A(M) \text { with }\|y\| \leq \delta \\
\text { it holds }\left\|A^{-1} y\right\|<\varepsilon \Leftrightarrow \\
\forall \varepsilon>0 \exists \bar{\delta}>0 \text { such that } \forall y \in A(M) \text { with }\|y\|<\bar{\delta} \text { it holds }\left\|A^{-1} y\right\|<\varepsilon,
\end{gathered}
$$

which is nothing else than $A^{-1}: A(M) \rightarrow X$ is continuous at zero.

We summarize the preceding discussion.

Definition 3. We call the problem (1) conditionally well-posed on $M$ if the mapping $A^{-1}$ restricted to $A(M)$ is continuous.

A famous theorem by A. N. Tikhonov, see [10], asserts that the problem is conditionally well-posed whenever the set $M \subset X$ is compact. For some further discussion and examples concerning conditional well-posedness we also refer to [4, 15, 13].

Theorem 1. Suppose that the set $M \subseteq X$ is star-shaped at zero. Then the following assertions are equivalent:

(i) The problem (1) is conditionally well-posed on $M$.

(ii) The modulus of continuity $\omega_{M}$ is right-continuous at zero.

(iii) The modulus of continuity $\omega_{M}$ is continuous on $[0,+\infty)$.

Proof. The equivalence of the first two assertions is a consequence of Proposition 4. The equivalence of the last two assertions follows from Corollary 1.

Tikhonov's result translates to the following statement.

Proposition 5. If the set $M \subset X$ is compact and star-shaped at zero, then $\omega_{M}(\delta) \rightarrow 0$ as $\delta \rightarrow 0$, and hence the modulus of continuity $\omega_{M}$ is continuous on $[0,+\infty)$. 
We present the following examples.

Example 1. Suppose that the operator $A$ is injective and has a non-closed range. By denoting with $B_{X}:=\{x \in X:\|x\| \leq 1\}$ the closed unit ball of $X$, we show that for all $\delta>0$ it holds

$$
\omega_{B_{X}}(\delta)=\sup \{\|x\|:\|x\| \leq 1,\|A x\| \leq \delta\}=1 .
$$

Indeed, consider a fixed $\delta>0$. Obviously, $\omega_{B_{X}}(\delta) \leq 1$. Since the range of $A$ is non-closed, there is no $K>0$ such that $\|x\| \leq K\|A x\|$ for all $x \in X$. Thus there exists a sequence $\left\{\tilde{x}_{n}\right\}_{n \geq 1} \subset X \backslash\{0\}$ such that $\frac{\left\|\tilde{x}_{n}\right\|}{\left\|\tilde{A} x_{n}\right\|} \rightarrow+\infty$ as $n \rightarrow+\infty$. By defining for all $n \geq 1$ $x_{n}:=\frac{1}{\left\|\tilde{x}_{n}\right\|} \tilde{x}_{n}$, one has that $\left\|x_{n}\right\|=1$ and $\left\|A x_{n}\right\| \rightarrow 0$ as $n \rightarrow+\infty$. Thus there exists $n(\delta) \geq 1$ such that $\left\|A x_{n}\right\| \leq \delta$ for all $n \geq n(\delta)$ and this provides the desired assertion. Consequently, the modulus of continuity $\omega_{M}$ is not right-continuous at zero for $M=B_{X}$.

Example 2. If $\{0\} \neq L \subset X$ is a finite-dimensional linear subspace then we have $\omega_{M}(\delta) \asymp \delta$ as $\delta \rightarrow 0$ for $M=L$. Indeed, the image space $A(L)$ is a closed linear subspace in $Y$, and therefore $A^{-1}: A(L) \rightarrow L$ is a bounded linear operator. Thus there is a constant $C<+\infty$ for which $\|x\| \leq C\|A x\|, x \in L$. But this yields that $\omega_{L}(\delta) \leq C \delta$ for all $\delta>0$. Next, if $\omega_{L}$ would vanish, say at $\delta_{0}>0$, then $\left\{x \in L:\|A x\| \leq \delta_{0}\right\}=\{0\}$, which is not true. In the light of Corollary 2 the above assertion is proved.

2.4. Regularizability. We now highlight the problem of the continuity of the modulus of continuity to the regularizability problem. To this end we introduce the local companion to the function $\Omega_{M}$ from (2), and we introduce, given any $x \in M$, the function

$$
\Omega_{M}^{l o c}(x, \delta):=\sup \left\{\left\|x-x^{\prime}\right\|: x^{\prime} \in M,\left\|A x-A x^{\prime}\right\| \leq \delta\right\} .
$$

Plainly, $\sup _{x \in M} \Omega_{M}^{l o c}(x, \delta)=\Omega_{M}(\delta)$.

In agreement with the usual nomenclature we call a reconstruction $S$ interpolatory if for each $x \in M$, and data $y^{\delta}$ with $\left\|A x-y^{\delta}\right\| \leq \delta$, we have that $S\left(y^{\delta}\right) \in M$ and $\left\|A S\left(y^{\delta}\right)-y^{\delta}\right\| \leq \delta$.

Lemma 3. Let $S$ be any reconstruction.

(i) If $S$ is interpolatory, then we have for each $x \in M$ that

$$
e(S, x, \delta) \leq \Omega_{M}^{l o c}(x, 2 \delta), \quad \delta>0 .
$$

(ii) If the set $M$ is centrally-symmetric, then

$$
\omega_{M}(\delta) \leq e(S, M, \delta), \quad \delta>0 .
$$

(iii) If the set $M$ is convex and centrally-symmetric, then for each $x \in M$ it holds

$$
\Omega_{M}(x, \delta) \leq 2 \omega_{M}(\delta / 2), \quad \delta>0
$$

Thus, for convex centrally-symmetric sets $M$ we have

$$
\omega_{M}(\delta) \leq e(S, M, \delta) \leq \Omega_{M}(2 \delta) \leq 2 \omega_{M}(\delta), \quad \delta>0,
$$

for any interpolatory reconstruction $S$. 
Proof. Given data $y^{\delta}$ and interpolatory reconstruction $S$ we let $x^{\prime}:=S\left(y^{\delta}\right) \in M$. Then $\left\|x-S\left(y^{\delta}\right)\right\| \leq \Omega_{M}^{l o c}(x, 2 \delta)$. Since this is true for any data $y^{\delta}$ with $\left\|A x-y^{\delta}\right\| \leq \delta$ the proof of the first assertion (ii) can be completed.

For the second assertion (ii) we argue as follows. For every $x \in M$, and due to symmetry also $-x \in M$ with $\|A x\|=\|A(-x)\| \leq \delta$ the data $y^{\delta}:=0$ are possible data, and we bound, using the triangle inequality

$$
e(S, M, \delta) \geq \max \{\|S(0)-x\|,\|S(0)+x\|\} \geq\|x\| .
$$

Therefore we conclude that

$$
e(S, M, \delta) \geq \sup \{\|x\|: x \in M,\|A x\| \leq \delta\}=\omega_{M}(\delta) .
$$

To prove item (iii) we fix any $x \in M$, and let $x^{\prime} \in M$ be arbitrary. Then $\hat{x}:=\left(x-x^{\prime}\right) / 2 \in$ $M$, and we have that $\|A \hat{x}\| \leq \delta / 2$, provided that $\left\|A x-A x^{\prime}\right\| \leq \delta$. Thus, $\left\|x-x^{\prime}\right\|=2\|\hat{x}\| \leq$ $2 \omega_{M}(\delta / 2)$. The bounds in $(6)$ are now easy consequences.

Remark 2. Indeed, interpolatory reconstructions always exist. To check this, fix $x \in M$ and data $y^{\delta}$ with $\left\|A x-y^{\delta}\right\| \leq \delta$ and consider the set $D\left(y^{\delta}\right):=\left\{z \in M:\left\|A z-y^{\delta}\right\| \leq \delta\right\}$. Since $x \in D\left(y^{\delta}\right)$ this set is non-empty, and any selection $S\left(y^{\delta}\right) \in D\left(y^{\delta}\right)$ will yield an interpolatory reconstruction. If the set $M \subset X$ is convex and compact, then there is even a continuous selection by Michael's continuous selection theorem, we refer to [2, $\S 7$ for details and extensions. For compact sets $M$ the construction of interpolatory reconstructions may be achieved by solving the optimization problem

$$
x_{q u}:=\arg \min _{z \in M}\left\|A z-y^{\delta}\right\|,
$$

which exists due to the compactness of $M$. It is readily checked that $x_{q u} \in M\left(y^{\delta}\right)$. This construction goes back to Ivanov [6] and it is called method of quasi-solutions, there.

Corollary 3. For convex centrally-symmetric sets $M$ the problem (1), is uniformly regularizable on $M$ if and only if the modulus of continuity $\omega_{M}$ is right-continuous at zero.

\section{Smoothness Classes in Hilbert space}

Here and in the subsequent section let $X$ and $Y$ be separable Hilbert spaces. We recall that the linear operator $A: X \rightarrow Y$ is assumed to be bounded and injective. For that case one can consider its self-adjoint companion $H:=A^{*} A$, where we set $a:=\|H\|=\|A\|^{2}$.

The typical smoothness classes as considered in inverse problems, and we mention source sets expressing general smoothness assumptions, and more recently, level sets, are based on the distribution function

$$
F_{x}^{2}(t):=\left\|\chi_{(0, t]}(H) x\right\|^{2}=\int_{0}^{t} d\left\|E_{\tau} x\right\|^{2}, \quad t \geq 0,
$$

which is well-defined and finite for each $x \in X$. Above, we let $\chi_{(0, t]}$ be the characteristic function of the interval $(0, t]$, and $E_{t}=E_{t}(H), 0 \leq t \leq a$, be the spectral resolution of the 
operator $H$. The following elementary properties are easily seen, for a further discussion and consequences cf. [3].

Lemma 4. Let $x \in X$ be arbitrary.

(i) The function $t \mapsto F_{x}(t)$ is right-continuous and non-decreasing.

(ii) If the operator $H$ in injective then $F_{x}(0)=0$.

(iii) For all $0<t \leq a$ we have that

$$
F_{\chi_{(0, t]}(H) x}(\tau)=F_{\chi_{(0, t]}(H) x}(t), \quad \tau \geq t .
$$

For a class $M \subset X$ we consider the associated function $\bar{F}_{M}$, given as

$$
\bar{F}_{M}(t):=\sup _{x \in M} F_{x}(t), \quad t \geq 0 .
$$

This function is finite whenever $M$ is bounded, and we thus will assume boundedness of $M$, throughout. It is also non-decreasing, and we have $\bar{F}_{M}(0)=0$. As it will turn out, the right-continuity of the function $\bar{F}_{M}(t)$ at zero and the right-continuity of the function $\omega_{M}$ at zero are closely related, and we will dwell into this, now. Best results are obtained for smoothness which is expressed through sets $M$, which are determined in the vicinity of zero of the distribution function $F_{x}(t), t>0$, only.

Definition 4 (spectral smoothness). We call a smoothness class $M$ spectral, if for each $t>0$ we have that $x \in M$ yields that $\chi_{(0, t]}(H) x \in M$.

Definition 5 (index function). We call a function $\varphi:(0, a] \rightarrow(0,+\infty)$ index function if it is continuous and increasing with $\lim _{t \searrow 0} \varphi(t)=0$.

Example 3. For an index function $\varphi$ we assign the smoothness class $\mathcal{M}_{\varphi}$ as

$$
\mathcal{M}_{\varphi}:=\{x=\varphi(H) v, \quad\|v\| \leq 1\},
$$

i.e., the image of the unit ball under the mapping $\varphi(H)$. Such classes are spectral since with $x=\varphi(H) v \in \mathcal{M}_{\varphi}$ we also have that

$$
\chi_{(0, t]}(H) x=\chi_{(0, t]}(H) \varphi(H) v=\varphi(H) \chi_{(0, t]}(H) v,
$$

and $\left\|\chi_{(0, t]}(H) v\right\| \leq\|v\| \leq 1$. We mention that

$$
\bar{F}_{\mathcal{M}_{\varphi}}(t)=\left\|\chi_{(0, t]}(H) \varphi(H)\right\| \leq \sup _{0<s \leq t} \varphi(s)=\varphi(t), \quad 0<t \leq a,
$$

such that $\bar{F}_{\mathcal{M}_{\varphi}}$ is right-continuous at zero exactly if $\varphi$ was an index function.

Example 4. For an index function $\varphi$ we assign the level set $\mathcal{E}_{\varphi}$ as

$$
\mathcal{E}_{\varphi}:=\left\{x \in X: F_{x}(t) \leq \varphi(t), 0<t \leq a\right\} .
$$

In view of Lemma 4 iii) such classes also constitute spectral smoothness classes, and $\bar{F}_{\mathcal{E}_{\varphi}}$ is right-continuous at zero for index functions $\varphi$. 
Proposition 6. Suppose that the set $M \subset X$ is bounded. If the function $\bar{F}_{M}$ is rightcontinuous at zero then $\omega_{M}$ is also right-continuous at zero. Moreover, for spectral smoothness classes $M$ the converse also holds true.

Proof. To prove the first assertion we observe that for $0<t<a$ we can estimate the norm square as

$$
\|x\|^{2}=\int_{0}^{t} d F_{x}^{2}(\tau)+\int_{t}^{a} d F_{x}^{2}(\tau) \leq F_{x}^{2}(t)+\frac{1}{t} \int_{t}^{a} \tau d F_{x}^{2}(\tau) .
$$

From this it follows $\|x\|^{2} \leq F_{x}^{2}(t)+\frac{1}{t}\|A x\|^{2}$ and taking the supremum over the set $\{x \in$ $M:\|A x\| \leq \delta\}$ we obtain for $\delta>0$ and all sufficiently small $t>0$

$$
\omega_{M}^{2}(\delta) \leq \bar{F}_{M}^{2}(t)+\frac{\delta^{2}}{t} .
$$

By setting $t:=\delta$ and under the condition $\bar{F}_{M}(t) \rightarrow 0$ as $t \searrow 0$ the upper bound of

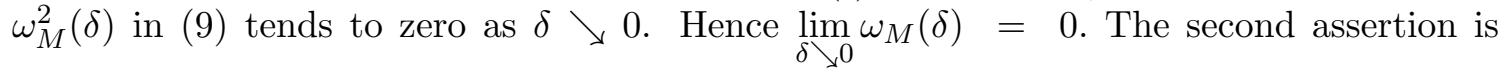
proved by contraposition. Without loss of generality we assume that $M$ belongs to the unit ball in $X$. Suppose that there is some $\varepsilon>0$ such that for all $t>0$ we have that $\bar{F}_{M}(t)=\sup _{x \in M}\left\|\chi_{(0, t]}(H) x\right\|>\varepsilon$. Thus we can find $\tilde{x}_{t} \in M$ with $\left\|\chi_{(0, t]}(H) \tilde{x}_{t}\right\| \geq \varepsilon$. We assign $x_{t}:=\chi_{(0, t]}(H) \tilde{x}_{t}, t>0$, and $x_{t} \in M$ since the set $M$ was assumed to be spectral. We thus have that for this $\varepsilon>0$ we can find a family $x_{t} \in M, t>0$ with $\left\|x_{t}\right\| \geq \varepsilon$. We claim that $\left\|A x_{t}\right\| \leq t$. Indeed, taking into account item (iii) of Lemma 4 we bound

$$
\left\|A x_{t}\right\|^{2}=\int_{0}^{a} \tau d F_{x_{t}}^{2}(\tau)=\int_{0}^{t} \tau d F_{x_{t}}^{2}(\tau) \leq t \int_{0}^{t} d F_{x_{t}}^{2}(\tau) \leq t\left\|x_{t}\right\|^{2} \leq t .
$$

Consequently we see that $\omega_{M}(\sqrt{t}) \geq \varepsilon, t>0$, which is a contradiction. The proof is complete.

Remark 3. Tight bounds for the modulus of continuity $\omega_{\mathcal{M}_{\varphi}}$ can be obtained under additional geometric (convexity) assumptions by means of general interpolation results within the framework of variable Hilbert scales, and we mention [12] for an early work on this. More recently this is pursued within the framework of conditionally stability estimates, see the recent study [13].

Evidently, Proposition 6 provides us with a characterization for the convex and centrallysymmetric smoothness classes $\mathcal{M}_{\varphi}$ and $\mathcal{E}_{\varphi}$.

In many cases the set $M$ is an ellipsoid in Hilbert space, i.e., there is an operator $G: Z \rightarrow X$, for a Hilbert space $Z$ such that

$$
M(G):=\{G v: \quad\|v\| \leq 1\},
$$

the image of the unit ball in $Z$ under the mapping $G$. Such ellipsoids $M(G)$ need not be spectral smoothness classes, in general. An obvious exception occurs for commuting operators $G$ and $H$. 
Example 5. In case that $G=\varphi(H): X \rightarrow X$ is a function of $H$, where $\varphi$ is any index function we have $M(G)=\mathcal{M}_{\varphi}$. Hence the concept of ellipsoidal sets generalizes general smoothness classes from Example 3.

For ellipsoids $M(G)$ we can rewrite

$$
\bar{F}_{M(G)}(t)=\sup _{\|v\| \leq 1}\left\|\chi_{(0, t]}(H) G v\right\|=\left\|\chi_{(0, t]}(H) G\right\|, \quad 0<t \leq a .
$$

where the latter is the operator norm of $\chi_{(0, t]}(H) G: Z \rightarrow X$.

The following was proved in [5, Thm. 4.4], and this shows that the bound in Lemma 3(iii) can be attained for ellipsoidal sets.

Theorem 2. For each ellipsoid $M(G)$ and each $\delta>0$ there is a linear reconstruction method $S_{\delta}$ such that

$$
e\left(S_{\delta}, M(G), \delta\right)=\omega_{M(G)}(\delta) .
$$

For compact operators $A$ we have the following characterization.

Proposition 7. Suppose that $A$ is a compact operator and $H:=A^{*} A$. The function $\bar{F}_{M(G)}$ is right-continuous at zero exactly if the operator $G$ is compact.

Proof. We notice that for a compact operator $H$ from above the operator $\chi_{(0, t]}(H)$ is a finite co-dimensional orthogonal projection, and hence it can be written as $\chi_{(0, t]}(H)=I-P_{N}$, where $P_{N}$ is the projection onto the finite dimensional space $H_{N}:=\operatorname{span}\left(\left\{u_{j}, s_{j}>t\right\}\right)$. Thus, we see that $\left.\sup _{x \in M(G)} F_{x}(t)=\|\left(I-P_{N}\right) G\right) \|$. As $t \searrow 0$ the dimension of the spaces $H_{N}$ will increase to $+\infty$. From this we conclude that $\sup _{x \in M(G)} F_{x}(t) \rightarrow 0$ if and only if the operator $G$ is approximable by finite rank operators, and thus compact.

\section{ON THE CONCAVITY OF THE MODUlus OF CONTINUITY}

Now, under the setting and notation of the previous section we (re)prove (see [4, Theorem]) the concavity of the function $\omega_{M}^{2}(\sqrt{\delta}), 0 \leq \delta<+\infty$, for $M=\mathcal{M}_{\varphi}$, and $M=\mathcal{E}_{\varphi}$ (for an index function $\varphi$, see Examples 3 and 4 , by using some tools of convex analysis.

For the beginning we notice that, according to the spectral theorem for bounded selfadjoint linear operators in Hilbert spaces (see [9, Chapt. VII]), there exist a measurable space $(\Omega, \mathcal{A}, \mu)$, a unitary transformation $U: X \rightarrow L^{2}(\Omega, \mathcal{A}, \mu)$ and a measurable function

$$
f: \Omega \rightarrow \sigma(H) \backslash\{0\} \subseteq(0,\|H\|] \subset \mathbb{R}
$$

such that $T_{f}:=U H U^{*}: L^{2}(\Omega, \mathcal{A}, \mu) \rightarrow L^{2}(\Omega, \mathcal{A}, \mu)$ is a multiplication operator defined as

$$
\left[T_{f} h\right](\omega):=f(\omega) h(\omega) \text { for all } \omega \in \Omega .
$$

By [4, Proposition 3] we get for an arbitrary set $M \subseteq X$ that

$$
\omega_{M}(\delta):=\omega_{M, A}(\delta)=\omega_{M, H^{\frac{1}{2}}}(\delta)=\omega_{U M, T_{\sqrt{f}}}(\delta) \text { for all } \delta \geq 0 .
$$

In the formula above the second lower index in the modulus of the continuity denotes the bounded linear operator to which this is associated, while $U M:=\{U x: x \in M\}$ stands for the image of the set $M$ through the operator $U$. 
For the images of the sets $\mathcal{M}_{\varphi}$ and $\mathcal{E}_{\varphi}$ through the unitary transformation $U$ we have, according to [4, Lemma 1], the representations

$$
U \mathcal{M}_{\varphi}=\left\{g \in L^{2}(\Omega, \mathcal{A}, \mu): g=\varphi(f) h,\|h\|_{L^{2}(\Omega, \mathcal{A}, \mu)} \leq 1\right\}
$$

and, respectively,

$$
U \mathcal{E}_{\varphi}=\left\{g \in L^{2}(\Omega, \mathcal{A}, \mu): \int_{0<f(\omega) \leq t} g^{2}(\omega) d \mu(\omega) \leq \psi^{2}(t) \forall t \in(0, a]\right\} .
$$

The main result of this section follows.

Theorem 3. Let $A: X \rightarrow Y$ be an injective and bounded linear operator with non-closed range $\mathcal{R}(A)$ mapping between separable Hilbert spaces $X$ and $Y$ and let $\varphi:\left(0,\|A\|^{2}\right] \rightarrow$ $(0,+\infty)$ be an arbitrary index function. Then the functions

$$
\delta \mapsto \omega_{\mathcal{M}_{\varphi}}^{2}(\sqrt{\delta})
$$

and

$$
\delta \mapsto \omega_{\mathcal{E}_{\varphi}}^{2}(\sqrt{\delta})
$$

are concave on the interval $[0,+\infty)$.

Proof. To prove that $\delta \mapsto \omega_{\mathcal{M}_{\varphi}}^{2}(\sqrt{\delta})$ is concave on $[0,+\infty)$, we let be $\Theta(t):=\sqrt{t} \varphi(t)$ for all $0<t \leq a$. According to (11) and by making use of the representation given for $U \mathcal{M}_{\varphi}$ above, it holds, for all $\delta \geq 0$,

$$
\begin{aligned}
-\omega_{\mathcal{M}_{\varphi}}^{2}(\sqrt{\delta}) & =\inf \left\{-\|g\|_{L^{2}(\Omega, \mathcal{A}, \mu)}^{2}: g=\varphi(f) h,\|h\|_{L^{2}(\Omega, \mathcal{A}, \mu)} \leq 1,\|\sqrt{f} g\|_{L^{2}(\Omega, \mathcal{A}, \mu)}^{2} \leq \delta\right\} \\
& =\inf \left\{-\|\varphi(f) h\|_{L^{2}(\Omega, \mathcal{A}, \mu)}^{2}:\|h\|_{L^{2}(\Omega, \mathcal{A}, \mu)}^{2} \leq 1,\|\Theta(f) h\|_{L^{2}(\Omega, \mathcal{A}, \mu)}^{2} \leq \delta\right\} .
\end{aligned}
$$

It will be convenient to consider the duality pairing $\langle\cdot, \cdot\rangle$ between $L^{\infty}(\Omega, \mathcal{A}, \mu)$ and $L^{1}(\Omega, \mathcal{A}, \mu)$. Because both the functions $\varphi(f)$ and $\Theta(f)$ are uniformly bounded, and the function $k:=h^{2} \geq 0$ belongs to $L^{1}(\Omega, \mathcal{A}, \mu)$, the representation 12$)$ rewrites as

$$
-\omega_{\mathcal{M}_{\varphi}}^{2}(\sqrt{\delta})=\inf \left\{\left\langle-\varphi^{2}(f), k\right\rangle: \quad\langle 1, k\rangle \leq 1,\left\langle\Theta^{2}(f), k\right\rangle \leq \delta, k \geq 0\right\}
$$

which results in

$$
-\omega_{\mathcal{M}_{\varphi}}^{2}(\sqrt{\delta})=\inf _{(k, \delta) \in L^{1}(\Omega, \mathcal{A}, \mu) \times \mathbb{R}} \Psi(k, \delta),
$$

where the function $\Psi: L^{1}(\Omega, \mathcal{A}, \mu) \times \mathbb{R} \rightarrow \mathbb{R} \cup\{+\infty\}$ is given as

$$
\Psi(k, \delta)= \begin{cases}\left\langle-\varphi^{2}(f), k\right\rangle, & \langle 1, k\rangle \leq 1,\left\langle\Theta^{2}(f), k\right\rangle \leq \delta, k \geq 0, \delta \geq 0, \\ +\infty, & \text { otherwise. }\end{cases}
$$

The function $\Psi$ is a convex function in both variables, and this fact implies the convexity of marginal function $\delta \mapsto-\omega_{\mathcal{M}_{\varphi}}^{2}(\sqrt{\delta})$ (cf. [14, Theorem 2.1.3 (v)]), which further yields the concavity of $\omega_{\mathcal{M}_{\varphi}}^{2}(\sqrt{\cdot})$ on $[0,+\infty)$, and which completes the proof. The proof of the the concavity of $\delta \mapsto \omega_{\mathcal{E}_{\varphi}}^{2}(\sqrt{\delta})$ is similar and hence omitted. 


\section{REFERENCES}

[1] A. Bakushinsky and A. Goncharsky. Ill-Posed Problems: Theory and Applications, volume 301 of Mathematics and its Applications. Kluwer Academic Publishers Group, Dordrecht, 1994.

[2] C. Bessaga and A. Pełczyński. Selected Topics in Infinite-Dimensional Topology, volume 58 of Monogr. Mat. PWN-Polish Sci. Publ., Warsaw, 1975.

[3] J. Flemming, B. Hofmann, and P. Mathé. Sharp converse results for the regularization error using distance functions. Inverse Problems 27(2): 025006 (18pp), 2011.

[4] B. Hofmann and P. Mathé. Some note on the modulus of continuity for ill-posed problems in Hilbert space. Preprint 2011-7, Preprint Series of Faculty of Mathematics, Chemnitz University of Technology, Chemnitz, 2011. Electronically available at http://www.tu-chemnitz.de/mathematik/preprint/2011/PREPRINT_07.pdf

[5] B. Hofmann, P. Mathé, and M. Schieck. Modulus of continuity for conditionally stable ill-posed problems in Hilbert space. J. Inverse Ill-Posed Probl., 16(6):567-585, 2008.

[6] V. K. Ivanov. Approximate solution of operator equations of the first kind. Ž. Vyčisl. Mat. i Mat. Fiz., 6:1089-1094, 1966.

[7] V. K. Ivanov, V. V. Vasin, and V. P. Tanana. Theory of Linear Ill-Posed Problems and its Applications. Inverse and Ill-posed Problems Series. VSP, Utrecht, second edition, 2002. Translated and revised from the 1978 Russian original ["Nauka", Moscow].

[8] N. Korneřchuk. Exact Constants in Approximation Theory, volume 38 of Encyclopedia of Mathematics and its Applications. Cambridge University Press, Cambridge, 1991.

[9] M. Reed and B. Simon. Methods of Modern Mathematical Physics. I - Functional Analysis (2nd. Ed.). Academic Press, Inc. [Harcourt Brace Jovanovich, Publishers], New York, 1980.

[10] A. N. Tikhonov. On the stability of inverse problems. C. R. (Doklady) Acad. Sci. URSS (N.S.), 39:176$179,1943$.

[11] V. A. Vinokurov. Regularization by continuous mappings. Ž. Vyčisl. Mat. i Mat. Fiz., 11:1584-1586, 1971.

[12] U. Tautenhahn. Optimality for ill-posed problems under general source conditions.. Numer. Funct. Anal. Optim., 19, no. 3-4, 377-398, 1998.

[13] U. Tautenhhn, U. Hämarik, B. Hofmann, and Y. Shao. Conditional stability estimates for ill-posed PDE problems by using interpolation. Preprint 2011-16, Preprint Series of Faculty of Mathematics, Chemnitz University of Technology, Chemnitz, 2011. Electronically available at http://www.tu-chemnitz.de/mathematik/preprint/2011/PREPRINT_16.pdf

[14] C. Zalinescu. Convex Analysis in General Vector Spaces. World Scientific, Singapore, 2002.

Department of Mathematics, Chemnitz University of Technology, 09107 Chemnitz, GerMANY

E-mail address: bot@mathematik.tu-chemnitz.de, hofmannb@mathematik.tu-chemnitz.de

Weierstrass Institute for Applied Analysis and Stochastics, Mohrenstrasse 39, 10117 Berlin, Germany

E-mail address: mathe@wias-berlin.de 